\title{
Generation of pancreatic islet cells and progenitor cells from embryonic stem cells
}

\author{
Wei Jiang ${ }^{1}$, Song Chen ${ }^{1}$, Yan Shi ${ }^{1}$, Mingxiao Ding ${ }^{1}$, Hongkui Deng ${ }^{1}$ \\ ${ }^{I}$ Department of Cell Biology and Genetics, College of Life Sciences, Peking University, China
}

Our lab recently established an effective method for inducing the differentiation of human ES cells into insulinproducing cells using a serum-free system. The protocol is designed to mimic pancreatic islet development and includes three developmental events: definitive endoderm formation, pancreas specification and endocrinal islet maturation. We show that differentiated cells express islet specific markers such as C-peptide, pdx1, insulin, glucagon and glut2. Among the differentiated cells, we observed islet-like cluster structures and most of the cells in these clusters co-expressed C-peptide and pdx1. In the islet-like clusters, there were a few pdx1 and somatostatin co-expressing cells; however, we did not find any examples of cells that co-expressed pdx1 with either glucagon or amylase. The differentiated cells were also able to release insulin and $\mathrm{C}$-peptide in response to glucose stimuli. When transplanted into renal capsules, the differentiated human ES cells maintained C-peptide expression and rescued hyperglycemia in STZ induced diabetic nude mice. Our findings offer a promising in vitro model for studying the mechanism of pancreatic islet cell and pancreatic stem/progenitor cell differentiation.

Keyword: human embryonic stem cells; directed differentiation; insulin-producing cell

Cell Research (2008) 18:s28. doi: 10.1038/cr.2008.118; published online 4 August 2008

Correspondence: Hongkui Deng

E-mail: hongkui_deng@pku.edu.cn

Hongkui Deng, $\mathrm{PhD}$ is a Professor of Cell Biology at Peking University and Associate Director of Chemical Genomics Laboratory at Peking University. He is also a Cheung Kong scholar. Prior to Peking University,
Hongkui was the Director of Molecular Biology at Viacell Inc. From 1995 to 1997 he was an Aaron Diamond Postdoctoral fellow at the NYU School of Medicine's Skirball Institute. He obtained his PhD of Immunology in 1995 from UCLA. Dr Deng's research focuses on human embryonic stem cells and animal models for human diseases 